\title{
Study of Heterosis Using Wild Abortive (WA) CMS Lines on Yield, Quality and Drought Related Traits in Rice (Oryza sativa L.)
}

\author{
S.K. Singh, Ravi Sahu, D.K. Singh and Prudhvi Raj Vennela* \\ Department of Genetics and Plant Breeding, Institute of Agricultural Sciences, \\ Banaras Hindu University, Varanasi (U.P.), 221005, India \\ *Corresponding author
}

\section{A B S T R A C T}

\begin{tabular}{l} 
Ke y w or d s \\
Heterosis, \\
Wild abortive \\
CMS lines, \\
$\begin{array}{l}\text { Hybrid, Drought, } \\
\text { SH (standard } \\
\text { hybrid), SV } \\
\text { (standard variety). }\end{array}$ \\
\hline Article Info \\
\hline $\begin{array}{l}\text { Accepted: } \\
\text { 23 June 2017 } \\
\text { Available Online: } \\
\text { 10 July 2017 }\end{array}$ \\
\hline
\end{tabular}

Keywords

Heterosis

Hybrid, Drought,

(standard variety)

To study the heterosis using wild abortive(WA) CMS lines on yield, quality and drought related traits in rice, 56 experimental hybrids were produced using Three WA CMS lines (Pusa 6A, IR79156A and IR 68897A) and 31 male genotypes. The present study showed that superior performance for all characters was not expressed in a single hybrid combination. However, different cross combinations were found to be superior for various characters. The top two high yielding crosses estimated on the basis of significant standard heterosis over SH (Arize6444) and SV (NDR-97) are Pusa-6A x HUR-105 and Pusa-6A x Pantdhan-12. The hybrids Pusa-6A x IR-36 and Pusa6A x HUR-105 showed relatively significant desirable heterosis for the quality traits viz., hulling recovery, milling recovery, kernel length, kernel breadth, kernel length breadth ratio. The hybrids IR 79156A x Danteshwari and Pusa6A x NDR-359 exhibited significant standard heterosis in desired direction for quality aspects along with higher magnitude of standard heterosis for yield. The hybrids Pusa6A x NDR-359 and Pusa6A x Pantdhan-12 showed relatively higher significant desirable standard heterosis over SH (Arize-6444 Gold) for the drought traits viz., chlorophyll content (at 90 DAS) and proline content.

\section{Introduction}

Rice is one of the most important staple food crops of India (Singh et al., 2014). In March 2016, United State department of Agriculture (USDA) estimated that the world rice production 2015-2016 will be 471.09 million tons, around 1.59 million tons more than previous month's projection. The production of rice in China is 145,770,000 metric tons, while India's is $103,000,000$ metric tons. In India the rice production in different states is West Bengal (15023.68), U.P (14416), A.P and Telangana (11510). The production and productivity of rice became stagnant from past few years. Hybrid rice technology is one of the most practically feasible tools to break the yield barriers. In China, the first hybrid rice variety for commercial cultivation was released by Yuan Long Ping in 1976. India also started this programme in 1989 and released 65 hybrid rice varieties till 2014 (Singh et al., 2015). Hybrid rice offers an opportunity to boost the yield potential of rice with yield advantage of $15-20 \%$ over conventional high-yielding varieties (Dar et al., 2014). A major challenge in indica rice hybrid breeding is to ensure that the heterotic rice hybrids possess grain quality that is at least comparable, if not superior, to that of 
inbred check varieties grown by farmers. The grain quality of rice hybrids depends on the grain quality of parents. It is therefore important that only parents that show consumer acceptability are chosen to make hybrids.

Thus, identification of restorers among elite quality cultivars can serve as important tool for the development of better quality rice hybrids. In many instances biotic and Abiotic stresses (mainly drought) are the reason for lower yield in hybrids. Now there is a requirement of producing hybrids which shows stable performance even under drought conditions. Therefore, the present study was undertaken to assess the hybrids for per se performance and heterosis with respect to yield, quality traits and drought related traits involving CMS lines and restorer lines in rice.

\section{Materials and Methods}

The experimental hybrids(56) were developed during Kharif 2014 by using Three Wild Abortive (WA) CMS lines viz., Pusa 6A, IR79156A and IR 68897A crossed with 31 male genotypes. Only 56 cross combinations could be made perfectly. Hence, the experiment was conducted during Kharif 2015 with these 56 experimental hybrids grown along with parents and checks at the Agricultural Research Farm, Institute of Agricultural Sciences, BHU, Varanasi, in a single row of $3 \mathrm{~m}$ with spacing of $20 \times 15 \mathrm{~cm}^{2}$ between row to row and plant to plant respectively in R.B.D. with 3 replications. All the recommended agronomic practices were adopted to raise the crop under rainfed condition. No irrigation was provided to the crop after transplanting. In all the hybrids observations were recorded on five randomly selected plants for estimation of magnitude of heterosis with respect to 12 quantitative traits viz., Hulling recovery, milling recovery, kernel length, Kernel breadth, kernel L/B ratio, amylose content, canopy temperature depression (CTD), stomatal conductance, leaf area index, proline content, chlorophyll content and grain yield plant ${ }^{-1}$. The character means of each replication was subjected for analysis of variance (Panse and Sukhatme, 1967) and estimation of heterosis over better parent, standard variety and standard hybrid.

\section{Results and Discussion}

All crosses showed marked variations in the expression of Heterobelteosis and Standard heterosis for yield, quality and drought related traits. Heterobelteosis for grain yield/plant ranged from $-18.23 \%$ (IR-79156A x URG30 ) to $60.83 \%$ (Pusa- 6A x HUR-105), for Hulling recovery, milling recovery from $11.95 \%$ (IR-79156A x BPT-5204) to $2.67 \%$ (IR-79156A x URG-42), $-11.84 \%$ (IR79156A x BPT-5204) to $4.01 \%$ (Pusa-6A x HUR-105) respectively, kernel length, Kernel breadth, kernel L/B ratio from $-18.99 \%$ (IR79156A x BPT-5204) to $14.96 \%$ (Pusa-6A x Danteshwari), $-13.29 \%$ (IR-79156A x BPT5204) to $71.15 \%$ (Pusa-6A x Susksamarat), $36.39 \%$ (Pusa-6A x Susksamarat) to $7.23 \%$ (Pusa-6A x NDR-359) respectively, amylose content, canopy temp. depression (CTD), stomatal conductance from $-13.73 \%$ (IR79156A x Akshaydhan) to $2.43 \%$ (Pusa-6A x Susksamarat), $\quad-4.65 \% \quad$ (Pusa-6A $\quad \mathrm{X}$ Danteshwari) to $0.72 \%$ (Pusa-6A x URG-30), $-12.19 \%$ (Pusa-6A x Pantdhan-12) to $125.13 \%$ (Pusa-6A x URG-30) respectively, leaf area index, proline content and chlorophyll content ranged from $-37.02 \%$ (Pusa-6A x IR-36) to 72.74\% (Pusa-6A x HUR-105), -56.47\% (IR-79156A x BPT5204) to $66.02 \%$ (Pusa-6A x Susksamarat), $10.94 \%$ (IR-79156A x URG-30) to $10.05 \%$ (Pusa-6A x NDR-359) [at 60 DAS] and $14.13 \%$ (IR-79156A $\mathrm{x}$ IR-36) to $14.63 \%$ (Pusa-6A x NDR-359) [at 90 DAS] respectively (Fig. 1 and Tables 1 and 2). 
Table.1 ANOVA for analysis of yield, quality and drought related traits in rice

\begin{tabular}{|c|c|c|c|c|c|c|c|c|c|c|c|c|c|c|}
\hline \multirow{2}{*}{$\begin{array}{c}\text { Source of } \\
\text { Variations }\end{array}$} & \multirow[t]{2}{*}{ df } & \multirow{2}{*}{$\begin{array}{l}\text { Grain yield/ } \\
\text { plant (g) }\end{array}$} & \multirow{2}{*}{$\begin{array}{c}\text { Hulling } \\
\text { recovery } \\
\%\end{array}$} & \multirow{2}{*}{$\begin{array}{c}\text { Milling } \\
\text { recovery } \\
\%\end{array}$} & \multirow{2}{*}{$\begin{array}{c}\text { Kernel } \\
\text { length } \\
(\mathrm{mm})\end{array}$} & \multirow{2}{*}{$\begin{array}{c}\text { Kernel } \\
\text { breadth } \\
(\mathrm{mm})\end{array}$} & \multirow{2}{*}{$\begin{array}{c}\text { Kernel } \\
\text { L/B ratio }\end{array}$} & \multirow{2}{*}{$\begin{array}{c}\text { Amylose } \\
\text { content } \\
(\%)\end{array}$} & \multirow{2}{*}{\begin{tabular}{|c|} 
Canopy \\
temp. \\
depression \\
$(\text { CTD })^{\circ} \mathrm{C}$
\end{tabular}} & \multirow{2}{*}{\begin{tabular}{|c}
$\begin{array}{c}\text { Stomatal } \\
\text { conductance } \\
\left(\mathbf{m m o l} / \mathbf{m}^{2} / \mathrm{S}\right)\end{array}$ \\
\end{tabular}} & \multirow{2}{*}{$\begin{array}{l}\text { Leaf } \\
\text { area } \\
\text { index }\end{array}$} & \multirow{2}{*}{\begin{tabular}{|c|} 
Proline \\
content $(\mu \mathrm{mol} / \mathrm{g}$ \\
Fresh Weight $)$
\end{tabular}} & \multicolumn{2}{|c|}{ Chlorophyll content } \\
\hline & & & & & & & & & & & & & At 60 DAS & At 90 DAS \\
\hline Replicates & 2 & 5.97 & 1.44 & 1.30 & 0.001 & 0.00 & 0.002 & 0.11 & 4.23 & 4978.83 & 0.14 & 0.07 & 9.67 & 10.72 \\
\hline Treatments & 32 & $100.70 * * *$ & $20.19 * * *$ & $21.97 * * *$ & $0.97 * * *$ & $0.18 * * *$ & $0.86 * * *$ & $4.85 * * *$ & 1.01 & $92450.43 * * *$ & $3.35 * * *$ & $25.69 * * *$ & $13.62 * * *$ & $25.01 * * *$ \\
\hline Hybrids & 16 & $83.88^{* * *}$ & $23.88 * * *$ & $25.10 * * *$ & $0.73 * * *$ & $0.21 * * *$ & $0.76^{* * *}$ & $4.52 * * *$ & 1.26 & $85783.75 * * *$ & $4.35 * * *$ & $23.43 * * *$ & $8.67 * *$ & 10.08 \\
\hline Parents & 13 & $43.13 * * *$ & $16.93 * * *$ & $16.35 * * *$ & $1.06 * * *$ & $0.15 * * *$ & $0.63 * * *$ & $5.63 * * *$ & 0.60 & $74172.74 * * *$ & $1.21 * * *$ & $32.05 * * *$ & $16.18 * * *$ & $33.86 * * *$ \\
\hline Hybrids vs. Parents & 1 & $1193.78 * * *$ & $17.16^{*}$ & $38.47 * * *$ & $4.94 * * *$ & $0.22 * * *$ & $4.94 * * *$ & 0.14 & 1.03 & $462102.34 * * *$ & $15.41 * * *$ & $2.49 * * *$ & $38.30 * *$ & $124.67 * * *$ \\
\hline Checks & 1 & $10.64^{*}$ & $17.61 *$ & $37.70 * * *$ & 0.00 & $0.03 * *$ & $0.07 *$ & $1.13^{*}$ & 3.23 & 117964.20 *** & $6.47 * * *$ & $22.66 * * *$ & $14.41 *$ & $56.43 * *$ \\
\hline Checks vs. Hybrids & 1 & $324.59 * * *$ & $14.71 *$ & $23.57 * *$ & $1.81 * * *$ & $0.40 * * *$ & $3.75 * * *$ & $8.59 * * *$ & 0.14 & $120531.98 * * *$ & $0.67 * *$ & $3.78 * * *$ & $18.95^{*}$ & $43.04 *$ \\
\hline Checks vs. Parents & 1 & 1.75 & 3.31 & 3.38 & $0.07 * *$ & $0.16^{* * *}$ & $0.73 * * *$ & $7.38 * * *$ & 0.01 & 352.92 & $1.14 * * *$ & $7.17 * * *$ & $52.72 * * *$ & 1.34 \\
\hline Error & 64 & 2.53 & 2.68 & 2.53 & 0.006 & 0.003 & 0.01 & 0.27 & 2.06 & 2662.47 & 0.09 & 0.10 & 3.56 & 6.48 \\
\hline Total & 98 & 34.66 & 8.37 & 8.85 & 0.32 & 0.06 & 0.29 & 1.76 & 1.76 & 32028.26 & 1.16 & 8.46 & 6.97 & 12.62 \\
\hline
\end{tabular}

* Significant at $5 \%$ level, $* *$ significant at $1 \%$ level and $* * *$ significant at $0.1 \%$ level

Table.2 Estimates of per se performance, heterobeltosis and standard heterosis for yield, quality and drought related traits in 17 hybrids of rice

\begin{tabular}{|c|c|c|c|c|c|c|c|c|c|c|c|c|c|c|c|c|c|}
\hline \multirow[t]{2}{*}{ S. No. } & \multirow{2}{*}{\begin{tabular}{|l|} 
Characters \\
Crosses
\end{tabular}} & \multicolumn{4}{|c|}{ Hulling recovery } & \multicolumn{4}{|c|}{ Milling recovery } & \multicolumn{4}{|c|}{ Kernel length } & \multicolumn{4}{|c|}{ Kernel breadth } \\
\hline & & Mean & BP & NDR-97 & \begin{tabular}{|l} 
Arize \\
6444 \\
Gold \\
\end{tabular} & Mean & BP & NDR-97 & \begin{tabular}{|l} 
Arize \\
6444 \\
Gold \\
\end{tabular} & Mean & $\mathbf{B P}$ & NDR-97 & \begin{tabular}{|l} 
Arize \\
6444 \\
Gold \\
\end{tabular} & Mean & BP & NDR-97 & \begin{tabular}{|l} 
Arize \\
6444 \\
Gold \\
\end{tabular} \\
\hline 1 & IR-79156A x Akshaydhan & 78.62 & 1.93 & 2.23 & $6.99 * *$ & 70.67 & 2.78 & 1.54 & $9.43 * *$ & 7.00 & $-3.00 * *$ & $10.30^{* * *}$ & $10.13^{* * *}$ & 1.87 & $11.31^{* * *}$ & $-13.02 * *$ & $-6.81 * *$ \\
\hline 2 & IR-79156A x IR-36 & 73.49 & -2.64 & $-4.45^{*}$ & 0.00 & 65.76 & -2.43 & $-5.51 * *$ & 1.83 & 7.37 & $2.17 *$ & $16.18^{* * *}$ & $16.00 * *$ & 1.72 & 2.58 & $-19.84 * *$ & $-14.12 * *$ \\
\hline 3 & IR-79156A x URG-42 & 77.49 & 2.67 & 0.76 & $5.46 * *$ & 69.85 & 3.65 & 0.37 & $8.17 * *$ & 6.42 & $-11.04 * *$ & 1.16 & 1.00 & 1.56 & $-6.94 *$ & $-27.29 * *$ & $-22.09 * *$ \\
\hline 4 & IR-79156A x Danteshwari & 79.29 & 1.04 & 3.10 & $7.91 * *$ & 69.85 & -0.52 & 0.36 & $8.16^{* * *}$ & 7.13 & -1.20 & $12.35^{* * *}$ & 12.17 ** & 1.57 & $-6.75^{*}$ & $-27.13 * *$ & $-21.93 * *$ \\
\hline 5 & IR-79156A x URG-30 & 74.27 & -1.60 & -3.43 & 1.08 & 66.38 & -1.51 & $-4.62 *$ & 2.79 & 7.04 & $-2.36^{*}$ & $11.04 * *$ & $10.86^{* *}$ & 1.69 & 0.79 & $-21.24 * *$ & $-15.61 * *$ \\
\hline 6 & IR-79156A x BPT-5204 & 69.43 & $-11.95 * *$ & $-9.72 * *$ & $-5.51 * *$ & 62.49 & $-11.84 * *$ & $-10.20^{* *}$ & -3.23 & 5.84 & $-18.99 * *$ & $-7.88 * *$ & $-8.03 * *$ & 1.46 & $-13.29 * *$ & $-32.25 * *$ & $-27.41 * *$ \\
\hline 7 & Pusa-6A x Akshaydhan & 76.93 & -2.38 & 0.02 & $4.69 *$ & 69.93 & -1.17 & 0.48 & $8.28 * *$ & 6.35 & -1.81 & 0.05 & -0.10 & 1.88 & $20.73 * *$ & $-12.40 * *$ & $-6.15 *$ \\
\hline 8 & Pusa-6A x IR-36 & 79.48 & 0.87 & 3.35 & $8.17 * *$ & 73.41 & $3.75^{*}$ & $5.48 * *$ & 13.67 ** & 6.81 & $4.93 * *$ & $7.41 * *$ & $7.24 * *$ & 1.76 & $12.61 * *$ & $-18.29 * *$ & $-12.46^{* * *}$ \\
\hline 9 & Pusa-6A x URG-42 & 74.80 & $-5.07 * *$ & -2.74 & 1.80 & 67.56 & $-4.51 *$ & -2.92 & $4.62 *$ & 6.58 & 1.75 & $3.68 * *$ & $3.52 * *$ & 1.79 & $14.96^{* *}$ & $-16.59 * *$ & $-10.63 * *$ \\
\hline 10 & Pusa-6A x Danteshwari & 79.48 & 0.87 & 3.35 & $8.17 * *$ & 69.87 & -1.24 & 0.40 & $8.20 * *$ & 7.66 & $14.96 * *$ & $20.70 * *$ & $20.51 * *$ & 1.78 & $14.10^{* * *}$ & $-17.21 * *$ & $-11.30 * *$ \\
\hline 11 & Pusa-6A x URG-30 & 73.59 & $-6.62 * *$ & $-4.32^{*}$ & 0.14 & 65.46 & $-7.49 * *$ & $-5.94 * *$ & 1.36 & 6.20 & $-4.13 * *$ & $-2.31 *$ & $-2.47^{*}$ & 2.06 & $32.05^{* * *}$ & -4.19 & 2.66 \\
\hline 12 & Pusa-6A x HUR-105 & 79.13 & 0.42 & 2.89 & $7.69 * *$ & 73.59 & $4.01 *$ & $5.74 * *$ & $13.95^{* *}$ & 7.09 & $4.47 * *$ & $11.82^{* * *}$ & $11.65^{* * *}$ & 1.76 & $13.03^{* *}$ & $-17.98 * *$ & $-12.13 * *$ \\
\hline 13 & Pusa-6A x IET-22202 & 78.70 & -0.12 & 2.33 & $7.10^{* *}$ & 71.95 & 1.70 & 3.39 & $11.42 * *$ & 7.36 & -0.90 & $16.03^{* * *}$ & $15.84 * *$ & 1.78 & $14.10^{* *}$ & $-17.21 * *$ & $-11.30 * *$ \\
\hline 14 & Pusa-6A x Susksamarat & 77.22 & -2.00 & 0.41 & $5.09 * *$ & 70.70 & -0.07 & 1.59 & $9.48^{* *}$ & 7.05 & $5.85^{* * *}$ & $11.19^{* * *}$ & $11.02^{* * *}$ & 2.67 & $71.15^{* *}$ & $24.19 * *$ & $33.06^{* *}$ \\
\hline 15 & Pusa-6A x IR-64 & 77.00 & -2.28 & 0.12 & $4.79^{*}$ & 69.32 & -2.03 & -0.39 & $7.34 * *$ & 7.19 & 11.19 ** & $13.29 * *$ & $13.12 * *$ & 1.92 & 23.29 ** & $-10.54 * *$ & -4.15 \\
\hline 16 & Pusa-6A x NDR-359 & 78.64 & -0.21 & 2.25 & $7.01 * *$ & 68.88 & -2.64 & -1.02 & $6.66 * *$ & 7.42 & $11.63^{* *}$ & $16.97 * *$ & $16.79 * *$ & 1.67 & $7.05^{*}$ & $-22.33 * *$ & $-16.78 * *$ \\
\hline \multirow[t]{3}{*}{17} & Pusa-6A x Pantdhan-12 & 78.88 & 0.11 & 2.57 & $7.35^{* *} *$ & 70.42 & -0.48 & 1.18 & $9.04 * *$ & 7.29 & $5.86^{* * *}$ & $14.92 * *$ & $14.74 * *$ & 1.75 & $12.39^{* *}$ & $-18.45^{* *}$ & $-12.62 * *$ \\
\hline & Mean & 76.85 & -1.59 & -0.08 & 4.58 & 69.18 & -1.18 & -0.59 & 7.13 & 6.93 & 1.14 & 9.23 & 9.06 & 1.81 & 13.13 & -15.99 & -9.99 \\
\hline & Range & \begin{tabular}{|l}
69.43 \\
to \\
79.48 \\
\end{tabular} & $\begin{array}{l}-11.95 \text { to } \\
2.67\end{array}$ & $\begin{array}{l}-9.72 \text { to } \\
3.35\end{array}$ & $\begin{array}{l}-5.51 \text { to } \\
8.17\end{array}$ & $\begin{array}{l}62.49 \\
\text { to } \\
73.59 \\
\end{array}$ & $\begin{array}{l}-11.84 \text { to } \\
4.01\end{array}$ & $\mid \begin{array}{l}-10.20 \text { to } \\
5.74\end{array}$ & $\begin{array}{l}-3.23 \text { to } \\
13.95\end{array}$ & $\begin{array}{l}5.84 \text { to } \\
7.66\end{array}$ & $\begin{array}{l}-18.99 \text { to } \\
14.96\end{array}$ & $\begin{array}{l}-7.88 \text { to } \\
20.70\end{array}$ & $\begin{array}{l}-8.03 \text { to } \\
20.51\end{array}$ & $\begin{array}{l}1.46 \text { to } \\
2.67\end{array}$ & $\begin{array}{l}-13.29 \text { to } \\
71.15\end{array}$ & $\begin{array}{l}-32.25 \text { to } \\
24.19\end{array}$ & $\begin{array}{l}-27.41 \text { to } \\
33.06\end{array}$ \\
\hline
\end{tabular}


Int.J.Curr.Microbiol.App.Sci (2017) 6(7): 2631-2640

\begin{tabular}{|c|c|c|c|c|c|c|c|c|c|c|c|c|c|c|c|c|c|}
\hline \multirow[b]{2}{*}{ S. No. } & \multirow{2}{*}{$\begin{array}{c}\text { Characters } \\
\text { Crosses }\end{array}$} & \multicolumn{4}{|c|}{ Kernel L/B ratio } & \multicolumn{4}{|c|}{ Amylose content } & \multicolumn{4}{|c|}{ Canopy temp. depression (CTD) } & \multicolumn{4}{|c|}{ Stomatal conductance } \\
\hline & & Mean & BP & NDR-97 & $\begin{array}{l}\text { Arize } \\
\text { 6444 } \\
\text { Gold } \\
\end{array}$ & Mean & BP & NDR-97 & $\begin{array}{l}\text { Arize } \\
6444 \\
\text { Gold } \\
\end{array}$ & Mean & BP & NDR-97 & $\begin{array}{c}\text { Arize } \\
6444\end{array}$ & Mean & BP & NDR-97 & $\begin{array}{c}\text { Arize } \\
6444\end{array}$ \\
\hline 1 & IR-79156A x Akshaydhan & 3.75 & $-12.87 * *$ & $26.86^{* *}$ & $18.19 * *$ & 20.73 & $-13.73 * *$ & $-10.37 * *$ & $-13.61 * *$ & 31.33 & -3.29 & -4.37 & 0.11 & 533.67 & $45.25 * *$ & 9.61 & $158.52^{* * *}$ \\
\hline 2 & IR-79156A x IR-36 & 4.28 & -0.39 & $45.03 * *$ & $35.12 * *$ & 22.23 & $-7.49 * *$ & $-3.89 *$ & $-7.36^{* *}$ & 31.40 & -2.48 & -4.17 & 0.32 & 814.40 & $121.67 * *$ & $67.27 * *$ & $294.51 * *$ \\
\hline 3 & IR-79156A x URG-42 & 4.11 & -4.50 & $39.05 * *$ & $29.55 * *$ & 21.57 & $-10.26 * *$ & $-6.77 * *$ & $-10.14 * *$ & 31.27 & -2.70 & -4.58 & -0.11 & 423.13 & $51.57 * *$ & -13.09 & $104.97 * *$ \\
\hline 4 & IR-79156A x Danteshwari & 4.55 & $5.89 *$ & $54.18 * *$ & $43.64 * *$ & 24.33 & 1.25 & $5.19 * *$ & 1.39 & 32.50 & -1.42 & -0.81 & 3.83 & 419.07 & $119.48 * *$ & -13.93 & $103.00 * *$ \\
\hline 5 & IR-79156A x URG-30 & 4.17 & -3.10 & $41.08 * *$ & $31.44 * *$ & 21.27 & $-11.51^{* *}$ & $-8.07 * *$ & $-11.39 * *$ & 31.27 & -1.88 & -4.58 & -0.11 & 416.27 & $102.46 * *$ & -14.50 & $101.65^{* *}$ \\
\hline 6 & IR-79156A x BPT-5204 & 4.02 & $-6.59 * *$ & $36.00 * *$ & $26.71 * *$ & 23.23 & -3.33 & 0.43 & -3.19 & 30.97 & -2.82 & -5.49 & -1.06 & 336.10 & $105.06 * *$ & $-30.97 * *$ & $62.81 * *$ \\
\hline 7 & Pusa-6A x Akshaydhan & 3.37 & $-18.80 * *$ & $14.11 * *$ & $6.31 *$ & 22.90 & -1.72 & -1.01 & $-4.58^{*}$ & 32.63 & 0.10 & -0.41 & 4.26 & 886.30 & $98.89 * *$ & $82.04 * *$ & $329.34 * *$ \\
\hline 8 & Pusa-6A x IR-36 & 3.88 & $-6.43 * *$ & $31.49 * *$ & $22.50 * *$ & 20.17 & $-13.45 * *$ & $-12.82 * *$ & $-15.97 * *$ & 32.53 & -0.20 & $\begin{array}{c}-0.71 \\
\end{array}$ & 3.94 & 484.80 & $25.37 *$ & -0.42 & $134.85 * *$ \\
\hline 9 & Pusa-6A x URG-42 & 3.67 & $-11.57 * *$ & $24.27 * *$ & $15.77 * *$ & 21.80 & $-6.44 * *$ & $-5.76 * *$ & $-9.17 * *$ & 31.30 & -3.99 & -4.48 & 0.00 & 407.30 & $45.90 * *$ & -16.34 & $97.30^{* * *}$ \\
\hline 10 & Pusa-6A x Danteshwari & 4.30 & 3.69 & $45.71 * *$ & $35.75 * *$ & 23.60 & -0.42 & 2.02 & -1.67 & 31.43 & -4.65 & -4.07 & 0.43 & 373.87 & $95.81 * *$ & $-23.21 * *$ & $81.11^{* * *}$ \\
\hline 11 & Pusa-6A x URG-30 & 3.01 & $-27.55 * *$ & 1.81 & -5.15 & 20.90 & $-10.30 * *$ & $-9.65 * *$ & $-12.92 * *$ & 32.83 & 0.72 & 0.20 & 4.90 & 462.87 & $125.13^{* * *}$ & -4.93 & $124.22 * *$ \\
\hline 12 & Pusa-6A x HUR-105 & 4.03 & -2.97 & $36.34 * *$ & $27.02 * *$ & 23.00 & -3.09 & -0.58 & $-4.17 *$ & 32.37 & -0.72 & -1.22 & 3.41 & 537.73 & $81.44 * *$ & 10.45 & $160.49 * *$ \\
\hline 13 & Pusa-6A x IET-22202 & 4.14 & -0.16 & $40.29 * *$ & $30.70 * *$ & 22.40 & $-3.86^{*}$ & -3.17 & $-6.67 * *$ & 31.73 & -2.66 & -3.15 & 1.38 & 398.87 & $110.97 * *$ & $-18.07 *$ & $93.22^{* *}$ \\
\hline 14 & Pusa-6A x Susksamarat & 2.64 & $-36.39 * *$ & $-10.61 * *$ & $-16.72 * *$ & 23.87 & 2.43 & 3.17 & -0.56 & 32.47 & -0.41 & -0.92 & 3.73 & 782.43 & $34.52 * *$ & $60.71 * *$ & $279.02 * *$ \\
\hline 15 & Pusa-6A x IR-64 & 3.74 & $-9.88 * *$ & $26.64 * *$ & $17.98 * *$ & 20.93 & $-10.16^{* *}$ & $-9.51^{* * *}$ & $-12.78 * *$ & 31.23 & -4.19 & -4.68 & -0.21 & 327.10 & -9.00 & $-32.82 * *$ & $58.45 * *$ \\
\hline 16 & Pusa-6A x NDR-359 & 4.45 & $7.23 * *$ & $50.68 * *$ & $40.38 * *$ & 23.37 & 0.29 & 1.01 & -2.64 & 32.77 & 0.51 & 0.00 & 4.69 & 444.47 & $30.51 *$ & -8.71 & $115.31 * *$ \\
\hline \multirow[t]{3}{*}{17} & Pusa-6A x Pantdhan-12 & 4.16 & 0.24 & $40.86^{* * *}$ & $31.23 * *$ & 22.83 & $-4.99 * *$ & -1.30 & $-4.86 * *$ & 31.80 & -2.45 & -2.95 & 1.60 & 391.97 & -12.19 & $-19.49^{*}$ & $89.88^{* * *}$ \\
\hline & Mean & 3.90 & -7.30 & 31.99 & 22.97 & 22.30 & -5.69 & -3.59 & -7.08 & 31.87 & -1.91 & -2.73 & 1.83 & 496.49 & 68.99 & 1.98 & 140.51 \\
\hline & Range & $\begin{array}{c}2.64 \\
\text { to } \\
4.55 \\
\end{array}$ & $\begin{array}{c}-36.39 \text { to } \\
7.23\end{array}$ & $\begin{array}{c}-10.61 \text { to } \\
54.18\end{array}$ & $\begin{array}{c}-16.72 \text { to } \\
43.64\end{array}$ & $\begin{array}{c}20.17 \\
\text { to } \\
24.33 \\
\end{array}$ & $\begin{array}{c}-13.73 \text { to } \\
2.43\end{array}$ & $\begin{array}{c}-12.82 \text { to } \\
5.19\end{array}$ & $\begin{array}{c}-15.97 \text { to } \\
1.39\end{array}$ & $\begin{array}{c}30.97 \\
\text { to } \\
32.83 \\
\end{array}$ & $\begin{array}{l}-4.65 \\
\text { to } 0.72\end{array}$ & $\begin{array}{c}-5.49 \text { to } \\
0.20\end{array}$ & $\begin{array}{c}-1.06 \text { to } \\
4.90\end{array}$ & $\begin{array}{c}327.10 \\
\text { to } \\
886.30 \\
\end{array}$ & $\begin{array}{c}-12.19 \text { to } \\
125.13\end{array}$ & $\begin{array}{c}-32.82 \text { to } \\
82.04\end{array}$ & $\begin{array}{c}58.45 \text { to } \\
329.34\end{array}$ \\
\hline
\end{tabular}


Int.J.Curr.Microbiol.App.Sci (2017) 6(7): 2631-2640

Contd...

\begin{tabular}{|c|c|c|c|c|c|c|c|c|c|c|c|c|c|c|c|c|c|}
\hline \multirow[b]{3}{*}{ S. No. } & \multirow{3}{*}{$\begin{array}{c}\text { Characters } \\
\text { Crosses }\end{array}$} & \multirow{2}{*}{\multicolumn{4}{|c|}{ Leaf area index }} & \multirow{2}{*}{\multicolumn{4}{|c|}{ Proline content }} & \multicolumn{8}{|c|}{ Chlorophyll content } \\
\hline & & & & & & & & & & \multicolumn{4}{|c|}{ At 60 DAS } & \multicolumn{4}{|c|}{ At 90 DAS } \\
\hline & & Mean & $\mathbf{B P}$ & NDR-97 & $\begin{array}{c}\text { Arize } \\
\text { 6444 } \\
\text { Gold }\end{array}$ & Mean & $\mathbf{B P}$ & NDR-97 & $\begin{array}{c}\text { Arize } \\
\text { 6444 } \\
\text { Gold }\end{array}$ & Mean & $\mathbf{B P}$ & NDR-97 & $\begin{array}{c}\text { Arize } \\
6444 \\
\text { Gold }\end{array}$ & Mean & $\mathbf{B P}$ & $\begin{array}{l}\text { NDR- } \\
97\end{array}$ & $\begin{array}{c}\text { Arize } 6444 \\
\text { Gold }\end{array}$ \\
\hline 1 & IR-79156A x Akshaydhan & 1.84 & $-29.90 * *$ & 10.42 & $-50.89 * *$ & 14.59 & $-15.17 * *$ & $7.12 * *$ & $49.90^{* * *}$ & 37.23 & -3.79 & $-10.14 * *$ & -2.87 & 42.07 & 5.78 & 2.77 & $20.88^{* * *}$ \\
\hline 2 & IR-79156A x IR-36 & 2.12 & $-19.08^{*}$ & 27.45 & $-43.32 * *$ & 12.47 & $-27.52 * *$ & $-8.47^{* * *}$ & $28.08^{* *}$ & 39.03 & 0.86 & -5.79 & 1.83 & 39.30 & $-14.13^{* *}$ & -3.99 & $12.93^{*}$ \\
\hline 3 & IR-79156A x URG-42 & 1.78 & $-32.06 * *$ & 7.01 & $-52.41 * *$ & 8.56 & $-50.21 * *$ & $-37.13 * *$ & $-12.02 * *$ & 36.10 & -6.72 & $-12.87 * *$ & -5.83 & 38.00 & -4.44 & -7.17 & 9.20 \\
\hline 4 & IR-79156A x Danteshwari & 2.90 & 10.81 & $74.55^{* *}$ & $-22.37 * *$ & 15.52 & $-9.79 * *$ & $13.93^{* * *}$ & $59.42 * *$ & 40.70 & 3.12 & -1.77 & 6.17 & 42.47 & -0.78 & 3.75 & $22.03^{* *}$ \\
\hline 5 & IR-79156A x URG-30 & 2.60 & -0.64 & $56.51^{* *}$ & $-30.39 * *$ & 8.65 & $-49.73 * *$ & $-36.51 * *$ & $-11.16^{* *}$ & 34.47 & $-10.94 * *$ & $-16.81^{* *}$ & $-10.09^{*}$ & 40.23 & 1.17 & -1.71 & $15.61^{*}$ \\
\hline 6 & IR-79156A x BPT-5204 & 2.92 & 11.58 & $75.75^{* *}$ & $-21.84 * *$ & 7.49 & $-56.47 * *$ & $-45.03 * *$ & $-23.08 * *$ & 35.70 & -7.75 & $-13.84 * *$ & -6.87 & 40.13 & 0.92 & -1.95 & $15.33^{*}$ \\
\hline 7 & Pusa-6A x Akshaydhan & 2.08 & $-24.88^{* *}$ & 25.25 & $-44.30 * *$ & 11.49 & $10.20^{* * *}$ & $-15.64 * *$ & $18.05^{* * *}$ & 39.30 & 5.27 & -5.15 & 2.52 & 38.63 & -1.86 & -5.62 & 11.02 \\
\hline 8 & Pusa-6A x IR-36 & 1.75 & $-37.02 * *$ & 5.01 & $-53.30 * *$ & 12.49 & $9.78 * *$ & $-8.27 * *$ & $28.36^{* *}$ & 39.67 & 2.94 & -4.26 & 3.48 & 40.43 & $-11.65^{*}$ & -1.22 & $16.19^{* *}$ \\
\hline 9 & Pusa-6A x URG-42 & 3.13 & 12.74 & $87.98^{* *}$ & $-16.40^{*}$ & 12.61 & $35.12^{* *}$ & $-7.44 * *$ & $29.52^{* * *}$ & 38.77 & 6.31 & -6.44 & 1.13 & 40.50 & 5.84 & -1.06 & $16.38^{* * *}$ \\
\hline 10 & Pusa-6A x Danteshwari & 2.66 & -4.09 & $59.92^{* *}$ & $-28.88 * *$ & 12.45 & $-4.38^{*}$ & $-8.59^{* * *}$ & $27.91^{* *}$ & 36.10 & $-8.53 *$ & $-12.87 * *$ & -5.83 & 39.57 & -7.55 & -3.34 & $13.70^{*}$ \\
\hline 11 & Pusa-6A x URG-30 & 1.98 & $-28.73 * *$ & 18.84 & $-47.15^{* *}$ & 12.40 & $13.31^{* *}$ & $-8.93 * *$ & $27.43^{* * *}$ & 37.60 & 3.11 & $-9.25^{*}$ & -1.91 & 42.13 & 8.50 & 2.93 & 21.07 ** \\
\hline 12 & Pusa-6A x HUR-105 & 5.60 & $72.74 * *$ & $236.47 * *$ & $49.64 * *$ & 10.63 & $-32.20 * *$ & $-21.98 * *$ & $9.18^{* *}$ & 38.30 & 0.79 & $-7.56^{*}$ & -0.09 & 40.23 & 5.14 & -1.71 & $15.61^{*}$ \\
\hline 13 & Pusa-6A x IET-22202 & 5.37 & $52.99^{* *}$ & $222.85 * *$ & $43.58^{* * *}$ & 10.41 & $-40.86^{* * *}$ & $-23.54 * *$ & $6.99 *$ & 37.13 & -2.28 & $-10.38 * *$ & -3.13 & 37.33 & -2.44 & -8.79 & 7.28 \\
\hline 14 & Pusa-6A x Susksamarat & 3.10 & 11.90 & $86.57 * *$ & $-17.02 *$ & 15.49 & $66.02^{* *}$ & $13.73^{* * *}$ & $59.14^{* * *}$ & 38.97 & 6.86 & -5.95 & 1.65 & 41.30 & 4.56 & 0.90 & $18.68^{\text {** }}$ \\
\hline 15 & Pusa-6A x IR-64 & 3.99 & $43.87^{* * *}$ & $139.88^{* * *}$ & 6.68 & 14.44 & -3.20 & $6.00 * *$ & $48.32^{* *}$ & 38.87 & 0.43 & -6.19 & 1.39 & 43.17 & $12.80^{*}$ & 5.46 & $24.04 * *$ \\
\hline 16 & Pusa-6A x NDR-359 & 4.44 & $59.98^{* *}$ & $166.73 * *$ & $18.63^{* * *}$ & 17.46 & 2.07 & $28.19 * *$ & $79.38^{* *}$ & 40.13 & $10.05^{*}$ & -3.14 & 4.70 & 43.87 & $14.63^{* * *}$ & 7.17 & $26.05^{* *}$ \\
\hline \multirow[t]{3}{*}{17} & Pusa-6A x Pantdhan-12 & 3.67 & $32.21^{* *}$ & $120.44 * *$ & -1.96 & 15.64 & $46.82^{* * *}$ & $14.81^{* * *}$ & $60.65^{* * *}$ & 38.00 & 4.20 & $-8.29 *$ & -0.87 & 42.50 & 6.78 & 3.83 & $22.13^{* * *}$ \\
\hline & Mean & 3.05 & 7.79 & 83.63 & -18.34 & 12.52 & -6.25 & -8.10 & 28.59 & 38.00 & 0.23 & -8.28 & -0.86 & 40.70 & 1.37 & -0.57 & 16.95 \\
\hline & Range & $\begin{array}{c}1.75 \text { to } \\
5.60\end{array}$ & \begin{tabular}{|c|}
-37.02 to \\
72.74
\end{tabular} & $\begin{array}{l}5.01 \text { to } \\
236.47\end{array}$ & $\begin{array}{c}-53.30 \text { to } \\
49.64\end{array}$ & $\begin{array}{l}7.49 \text { to } \\
17.46\end{array}$ & $\begin{array}{c}-56.47 \text { to } \\
66.02\end{array}$ & $\begin{array}{c}-45.03 \text { to } \\
28.19\end{array}$ & \begin{tabular}{|c|}
-23.08 to \\
79.38
\end{tabular} & $\begin{array}{c}34.47 \\
\text { to } \\
40.70\end{array}$ & $\begin{array}{c}-10.94 \text { to } \\
10.05\end{array}$ & \begin{tabular}{|c|}
-16.81 to \\
-1.77
\end{tabular} & $\begin{array}{c}-10.09 \text { to } \\
6.17\end{array}$ & $\begin{array}{c}37.33 \text { to } \\
43.87\end{array}$ & $\begin{array}{c}-14.13 \text { to } \\
14.63\end{array}$ & $\begin{array}{c}-7.17 \text { to } \\
7.17\end{array}$ & 7.28 to 26.05 \\
\hline
\end{tabular}


Int.J.Curr.Microbiol.App.Sci (2017) 6(7): 2631-2640

\begin{tabular}{|c|c|c|c|c|c|}
\hline \multirow[t]{2}{*}{ S. No. } & \multirow{2}{*}{\begin{tabular}{|c|} 
Characters \\
Crosses \\
\end{tabular}} & \multicolumn{4}{|c|}{ Grain yield/ plant } \\
\hline & & Mean & BP & $\begin{array}{l}\text { NDR-97 } \\
\end{array}$ & Arize 6444 Gold \\
\hline 1 & IR-79156A x Akshaydhan & 31.26 & $40.19^{* *}$ & $94.67 * *$ & $66.98 * *$ \\
\hline 2 & IR-79156A x IR-36 & 22.18 & 3.77 & $38.11^{* *}$ & $18.46^{* * *}$ \\
\hline 3 & IR-79156A x URG-42 & 20.79 & -1.55 & $29.43^{* *}$ & 11.02 \\
\hline 4 & IR-79156A x Danteshwari & 20.31 & -3.82 & $26.44 * *$ & 8.46 \\
\hline 5 & IR-79156A x URG-30 & 17.26 & $-18.23 * *$ & 7.49 & -7.80 \\
\hline 6 & IR-79156A x BPT-5204 & 25.72 & $21.83^{* *}$ & $60.17^{* *}$ & $37.39 * *$ \\
\hline 7 & Pusa-6A x Akshaydhan & 27.37 & $22.72^{* *}$ & $70.40^{* *}$ & $46.16^{* *}$ \\
\hline 8 & Pusa-6A x IR-36 & 22.32 & 3.27 & $39.00^{* *}$ & $19.23^{* *}$ \\
\hline 9 & Pusa-6A x URG-42 & 20.78 & -3.89 & $29.37^{* *}$ & 10.97 \\
\hline 10 & Pusa-6A x Danteshwari & 22.49 & 4.04 & $40.04 * *$ & $20.12^{* *}$ \\
\hline 11 & Pusa-6A x URG-30 & 31.56 & $45.98^{* *}$ & $96.49^{* *}$ & $68.54^{* *}$ \\
\hline 12 & Pusa-6A x HUR-105 & 34.77 & $60.83^{* *}$ & $116.48^{* *}$ & $85.69^{* *}$ \\
\hline 13 & Pusa-6A x IET-22202 & 26.16 & $21.00^{* *}$ & $62.87 * *$ & $39.70^{* *}$ \\
\hline 14 & Pusa-6A x Susksamarat & 20.23 & -6.43 & $25.94 * *$ & 8.03 \\
\hline 15 & Pusa-6A x IR-64 & 22.73 & 5.15 & $41.53^{* *}$ & $21.40^{* *}$ \\
\hline 16 & Pusa-6A x NDR-359 & 27.44 & $21.75^{* *}$ & $70.88^{* *}$ & $46.57^{* *}$ \\
\hline 17 & Pusa-6A x Pantdhan-12 & 34.49 & $59.54 * *$ & $114.74 * *$ & $84.19^{* *}$ \\
\hline & Mean & 25.17 & 16.24 & 56.71 & 34.42 \\
\hline & Range & 17.26 to 34.77 & -18.23 to 60.83 & 7.49 to 116.48 & -7.80 to 85.69 \\
\hline
\end{tabular}


Fig.1 Graphical representation of per se performance of various traits

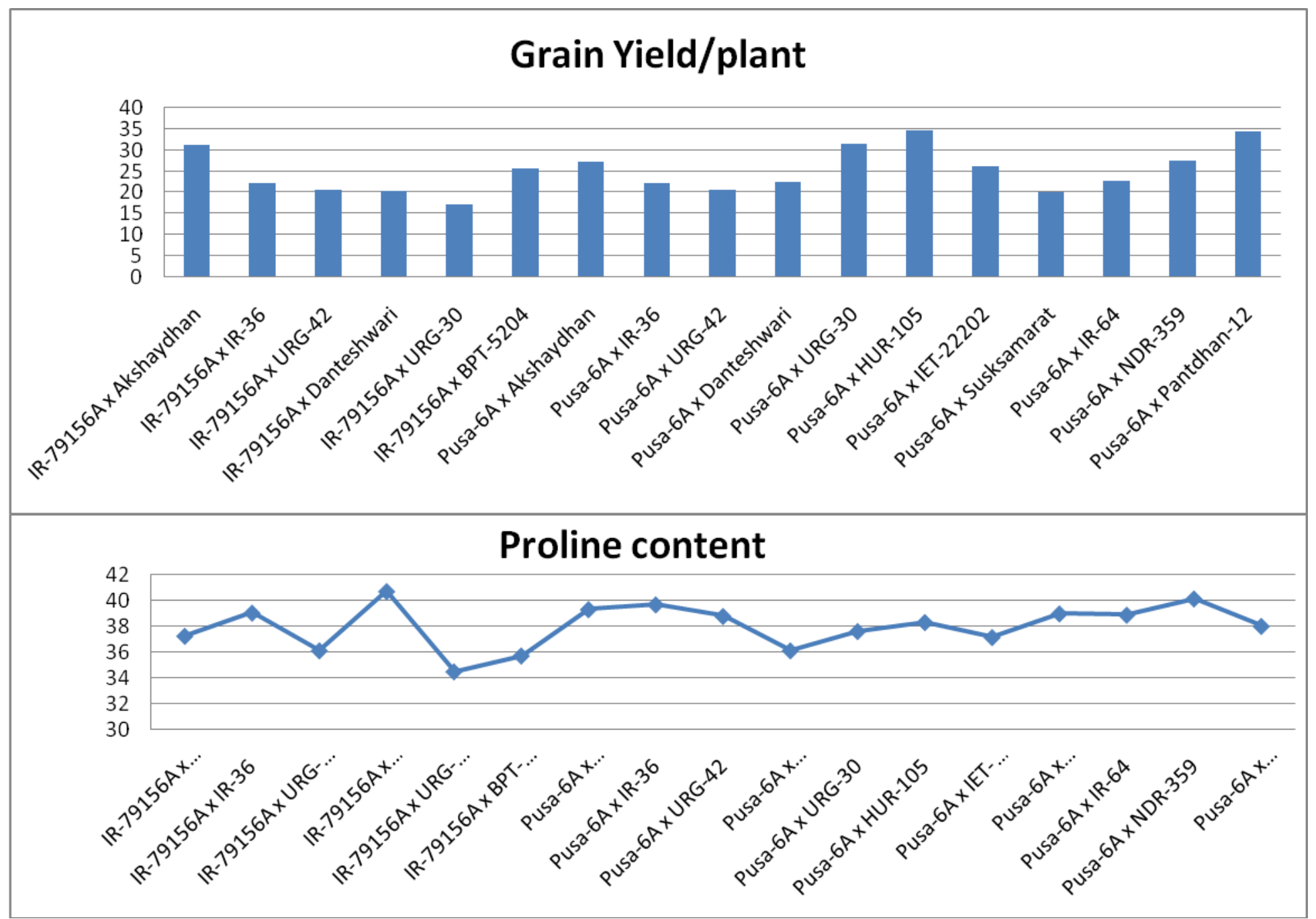




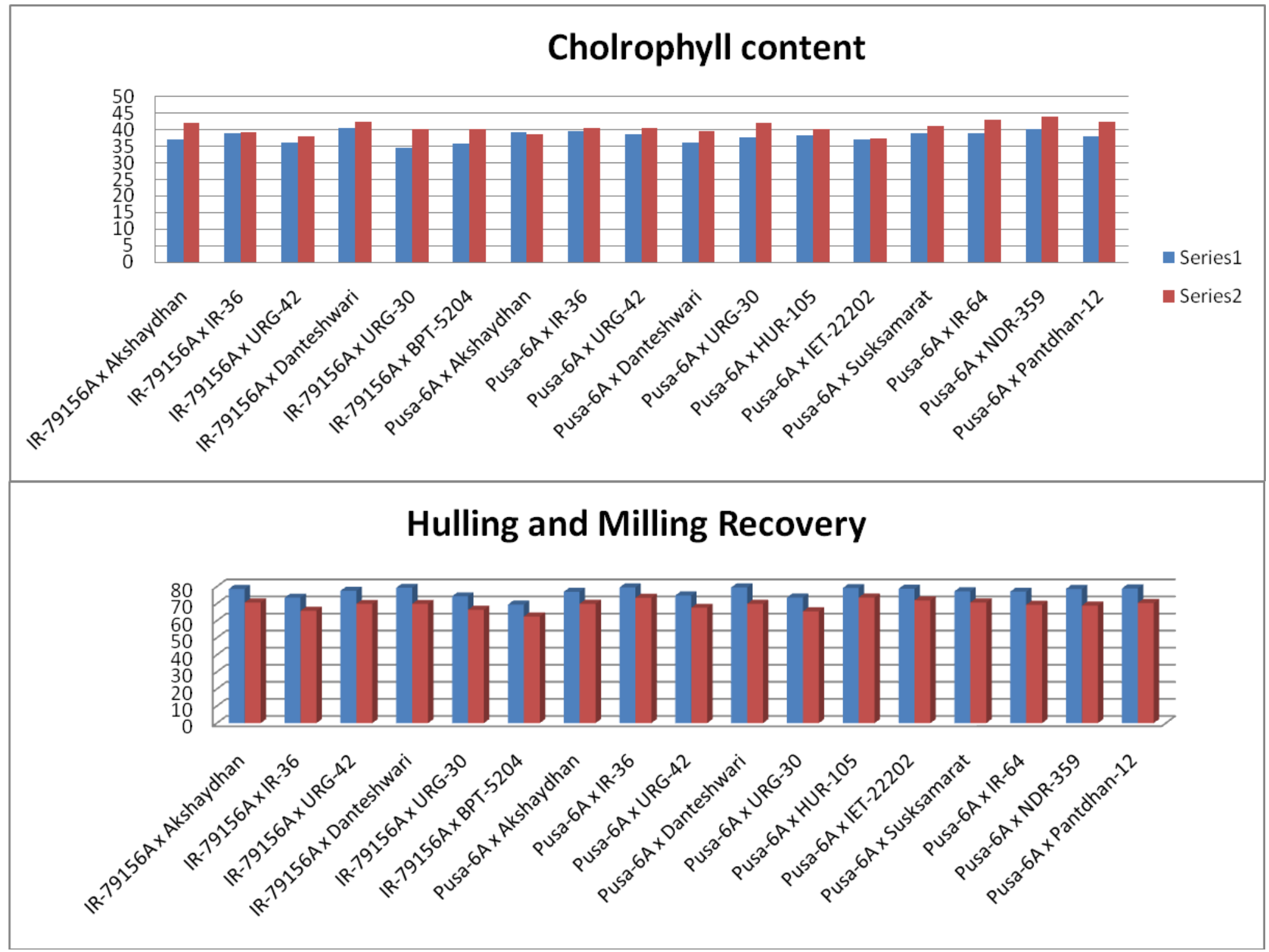


The standard heterosis over the best hybrid, Arize 6444 gold and over NDR-97 for grain yield per plant ranged from $-7.80 \%$ (IR79156A x URG-30) to $85.69 \%$ (Pusa-6A x HUR-105) and 7.49\% (IR-79156A x URG30) to $116.48 \%$ (Pusa-6A x HUR-105), for hulling recovery ranged from $-5.51 \%$ (IR79156A x BPT-5204) to $8.17 \%$ (Pusa-6A x IR-36 and Pusa-6A x Danteshwari) and 9.72\% (IR-79156A x BPT-5204) to $3.35 \%$ (Pusa-6A $x$ IR-36 and Pusa-6A $x$ Danteshwari), for milling recovery ranged from $-3.23 \%$ (IR-79156A x BPT-5204) to $13.95 \%$ (Pusa-6A x HUR-105) and $-10.20 \%$ (IR-79156A x BPT-5204) to 5.74\% (Pusa-6A $x$ HUR-105), for kernel length ranged from $8.03 \%$ (IR-79156A x BPT-5204) to $20.51 \%$ (Pusa-6A x Danteshwari) and $-7.88 \%$ (IR79156A x BPT-5204) to $20.70 \%$ (Pusa-6A x Danteshwari), for kernel breadth ranged from $-27.41 \%$ (IR-79156A x BPT-5204) to $33.06 \%$ (Pusa-6A x Susksamarat) and -32.25\% (IR79156A x BPT-5204) to $24.19 \%$ (Pusa-6A x Susksamarat), for kernel $\mathrm{L} / \mathrm{B}$ ratio ranged from $-16.72 \%$ (Pusa-6A x Susksamarat) to 43.64\% (IR-79156A x Danteshwari) and $10.61 \%$ (Pusa-6A x Susksamarat) to $54.18 \%$ (IR-79156A x Danteshwari), for amylose content ranged from $-15.97 \%$ (Pusa-6A x IR36) to $1.39 \%$ (IR-79156A x Danteshwari) and $-12.82 \%$ (Pusa-6A x IR-36) to $5.19 \%$ (IR79156A x Danteshwari), for canopy temp. depression (CTD) ranged from $-1.06 \%$ (IR$79156 \mathrm{~A} \times \mathrm{BPT}-5204)$ to $4.90 \%$ (Pusa-6A x URG-30) and $-5.49 \%$ (IR-79156A x BPT5204 ) to $0.20 \%$ (Pusa-6A x URG-30), for stomatal conductance ranged from $58.45 \%$ (Pusa-6A x IR-64) to $329.34 \%$ (Pusa-6A x Akshaydhan) and -32.82\% (Pusa-6A x IR-64) to $82.04 \%$ (Pusa-6A x Akshaydhan), for leaf area indexranged from-53.30\% (Pusa-6A x IR-36) to 49.64\% (Pusa-6A x HUR-105) and $5.01 \%$ (Pusa-6A x IR-36) to $236.47 \%$ (Pusa6A x HUR-105), for proline content ranged from $-23.08 \%$ (IR-79156A x BPT-5204) to $79.38 \%$ (Pusa-6A x NDR-359) and $-45.03 \%$
(IR-79156A x BPT-5204) to $28.19 \%$ (Pusa6A x NDR-359) and for chlorophyll content ranged from -10.09\% (IR-79156A x URG-30) to $6.17 \%$ (IR-79156A x Danteshwari) [at 60 DAS] and 7.28\% (Pusa-6A x IET-22202) to 26.05\% (Pusa-6A x NDR-359) [at 90 DAS] and $-16.81 \%$ (IR-79156A x URG-30) to $1.77 \%$ (IR-79156A x Danteshwari) [at 60 DAS] and $-7.17 \%$ (IR-79156A x URG-42) to 7.17\% (Pusa-6A x NDR-359)[at 90 DAS] respectively (Fig. 1). The higher heterotic effects among quality and drought related traits were observed for Kernel length, Kernel breadth, Kernel L/B ratio, Stomatal conductance, Proline content, Hulling recovery, Milling recovery and Chlorophyll content (at 90 days). The top two high yielding crosses on the basis of significant standard heterosis over SH(Arize6444) and SV(NDR-97) were Pusa-6A x HUR-105 (SH= 85.69\%, SV=116.48\%) and Pusa-6A $\mathrm{x}$ Pantdhan-12 $\quad(\mathrm{SH}=\quad 84.19 \%$, $\mathrm{SV}=114.74 \%$ ). Cross combinations Pusa-6A $\mathrm{x}$ IR-36 and Pusa6A x HUR-105 showed relatively significant desirable heterosis for the quality traits $v i z$, hulling recovery, milling recovery, kernel length, kernel breadth, kernel length breadth ratio. Hybrids IR 79156A x Danteshwari and Pusa6A x NDR-359 exhibited significant standard heterosis in desired direction for quality aspects along with higher magnitude of standard heterosis for yield. Cross combinations Pusa6A $\mathrm{x}$ NDR-359 and Pusa6A x Pantdhan-12 showed relatively higher significant desirable standard heterosis over SH (Arize-6444 Gold) for the drought traits viz., chlorophyll content (at 90 DAS) and proline content. Cross combinations Pusa 6A x HUR-105 and Pusa6A x IET-22202 showed significant desirable heterosis for the leaf area index. The present study revealed that superior performance for all characters was not expressed in a single hybrid combination. However, different cross combinations were found to be superior for various characters. 
These finding are in accordance with those of Binodh et al., (2006), Reddy et al., (2012),Venkanna et al., (2014), Babu et al., (2013), Singh et al., (2013) and Sharma et al., (2013), Priyanka et al., (2014).

\section{Acknowledgement}

Authors express their gratitude to UPCAR (Uttar Pradesh Council of Agricultural Research) for supporting this project.

\section{References}

Singh, Ashwinikumar, Singh, M.K., Prasad, S.K. and Pooja, S. 2014. Sequential herbicide application and nitrogen rates effectson weeds in direct seeded rice (Oryza sativa L.). The Ecoscan., 8(3\&4): 249-252.

Singh, S.K., Bhati, P.K., Amita, S. and Vikas, S. 2015. Super hybrid rice in China and India: current status and future prospects. Int. J. Agric. and Biol., 17(2): 221-232.

Dar, S.H., Rather, A.G., Sangher, G.S., Ahanger, M.A., Bhat, M.A., Ahmad, N., Syeed, T., Kurashi, S.N. and Ahmad, N. 2014. Identification of effective restorers and maintainers for development of rice hybrids in temperate ecology. Elect. J. Plant Bre., 5(4): 756-759.

Virmani, S.S., Virakamath, B.C., Laral, C.L., Toledo, R.S., Lopez, M.T. and Manalo, J.O. 1997. Hybrid Rice Breeding Manual, 151.

Panse, V.G. and Sukhatme, P.V. 1967. Statistical Methods for Agricultural Workers, 2nd Ed., Indian Council of Agricultural Research, New Delhi.

Lin, S.C. and Yuan, L.P. 1980. Hybrid rice breeding in China. Innovative approaches to rice breeding. IRRI, Manila, Philippines, pp. 35-51.

Yuan, L.P., Virmani, S.S. and Mao, C.X. 1989. Hybrid Rice-achievement and future outlook. In: 'Progress in irrigated rice research' $p p$. 219-235. IRRI, Manila, Philippines.

Binodh, A.K., Kalaiyarasi, R., Thiyagarajan, K. and Manonmani, S. 2006. Physiochemical cooking quality characteristics of promising varieties and hybrids in rice (Oryza sativa L.). Indian J. Genet., 66(2): 107-112.

Reddy, M.R., Raju, C.H.S., Srvani, T., Reddy, D., Reddy, N. 2012. Heterosis for yield and kernal size in aromatic rice (Oryza Sativa. L). Annals of Biol. Res., 3(6): 2662-2666.

Venkannan, V., Raju, C.H.S., Lingaiuh, N. and Roja, V.T. 2014. Studies on heterosis and inbreeding depression for grain yield and grain quality traits in rice (Oryza sativa L.). Int. J. Sci. Env. and Tech., 3(3): 910-916.

Babu, V.R., Shreya, K., Dangi, K.S., Usharani, G. and Nagesh, P. 2013. Evaluation of popular rice (Oryza sativa L.) hybrids for quantitative, qualitative and nutritional aspects. Int. J. Scientific and Res. Publications, 3(1): 1-8.

Singh, S.K., Vikash, S., Amita, S. and Pradeep, K.B. 2013. Heterosis for yield and yield components in rice (Oryza sativa L.). Bioinfolet, 10(2B): 752-761.

Sharma, S.K., Singh, S.K., Nandan, R., Amita, S., Kumar, V., Singh, M.K. 2013. Estimation of heterosis and inbreeding depression for yield and yield related traits in rice (Oryza sativaL.). Mol. Plant Bre., 29(4): 238-246.

Priyanka, K., Jaiswal, H.K. and Waza, S.A. 2014. Combining ability and heterosis for yield, its component traits and some grain quality parameters in rice (Oryza sativa L.). J. Appl. Natural Sci., 6(2): 495-506.

USDA, Rice Outlook. March 2016.

\section{How to cite this article:}

Singh, S.K., Ravi Sahu, D.K. Singh and Prudhvi Raj Vennela. 2017. Study of Heterosis Using Wild Abortive (WA) CMS Lines on Yield, Quality and Drought Related Traits in Rice (Oryza sativa L.). Int.J.Curr.Microbiol.App.Sci. 6(7): 2631-2640.

doi: https://doi.org/10.20546/ijcmas.2017.607.311 